\title{
VariantStore: an index for large-scale genomic variant search
}

\author{
Prashant Pandey, Yinjie Gao and Carl Kingsford* (D)
}

\author{
${ }^{*}$ Correspondence: \\ carlk@cs.cmu.edu \\ Computational Biology \\ Department, School of Computer \\ Science, Carnegie Mellon University, \\ Pittsburgh, USA
}

\begin{abstract}
Efficiently scaling genomic variant search indexes to thousands of samples is computationally challenging due to the presence of multiple coordinate systems to avoid reference biases. We present VariantStore, a system that indexes genomic variants from multiple samples using a variation graph and enables variant queries across any sample-specific coordinate system. We show the scalability of VariantStore by indexing genomic variants from the TCGA project in $4 \mathrm{~h}$ and the 1000 Genomes project in $3 \mathrm{~h}$. Querying for variants in a gene takes between 0.002 and 3 seconds using memory only $10 \%$ of the size of the full representation.
\end{abstract}

Keywords: Variation graph, Graph genomes, Pangenomes

\section{Background}

Advanced sequencing technology and computing resources have led to large-scale genomic sequencing efforts producing genomic variation data from thousands of samples, such as the 1000 Genomes project [1-3], GTEx [4], and The Cancer Genome Atlas (TCGA) [5]. Analysis of genomic variants combined with phenotypic information of samples promises to improve applications such as personalized medicine, population-level disease analysis, and cancer remission rate prediction. Although numerous studies [6-12] have been performed over the past decade involving genomic variation, the ability to scale these studies to large-scale data available today and in the near future is still limited.

On an individual sample, the typical result of sequencing, alignment, and variant calling is a collection of millions of sample-specific variants. A variant is identified by the position in the chromosome where it occurs, an alternative sequence, a list of samples that contain the variant, and phasing information. The standard file format to report these variants is the variant call file (VCF) [13].

Traditional reference-based variant storing methods, like VCF, store variants based on a single reference coordinate system and therefore have certain limitations. The choice of reference introduces reference bias in mapping, variant calling, and downstream analysis [14]. It leads to the misinterpretation of the "reference" as a baseline, while in fact it

(c) The Author(s). 2021 Open Access This article is licensed under a Creative Commons Attribution 4.0 International License which permits use, sharing, adaptation, distribution and reproduction in any medium or format, as long as you give appropriate credit to the original author(s) and the source, provide a link to the Creative Commons licence, and indicate if changes were made. The images or other third party material in this article are included in the article's Creative Commons licence, unless indicated otherwise in a credit line to the material. If material is not included in the article's Creative Commons licence and your intended use is not permitted by statutory regulation or exceeds the permitted use, you will need to obtain permission directly from the copyright holder. To view a copy of this licence, visit http://creativecommons.org/licenses/by/4.0/. The Creative Commons Public Domain Dedication waiver (http://creativecommons.org/publicdomain/zero/1.0/) applies to the data made available in this article, unless otherwise stated in a credit line to the data. 
is mostly a type specimen [14]. Storing highly variant sequence repertoires can be challenging because choosing a single representative sequence as reference is no longer viable and a bad choice of reference could lead to inefficient storage. For example, regions with high diversity, like T cell receptors (TCR), of which one person could have $10^{13}$ different clonotypes [15], are stored in a pan-genomic structure to preserve diversity.

A primary goal of pan-genomic variation analysis is to avoid biases that arise when treating a single genome as the reference when identifying or comparing variants across samples $[16,17]$. In pan-genomic variation analysis, we can identify and study variants based on any sample genome and compare variants from two samples directly without the need for a distinguished reference genome. The ability to compare samples directly also enables the comparison of variants derived from samples belonging to different species. Comparing variants from closely related species is often critical to study the evolutionary and mechanistic relationships between plant genomes that evolve quickly to adapt to external environments [18-21].

We present VariantStore, a system for efficiently indexing and querying genomic information (genomic variants and phasing information) from thousands of samples containing millions of variants. VariantStore supports querying variants occurring between two positions across a chromosome based on any sample coordinate system. Specifically, VariantStore supports queries of the following types:

- Find the closest variant to position $X$ in sample $S$ coordinates

- Find the sequence between positions $X$ and $Y$ for sample $S_{1}$ in sample $S_{2}$ coordinates

- Find all variants between positions $X$ and $Y$ for sample $S_{1}$ in sample $S_{2}$ coordinates

- Find all variants between positions $X$ and $Y$ in sample $S$ coordinates

The positions specified in variant queries are based on a coordinate system that uniquely identifies the positions of variants in a given genome [22]. Each sample in the input data can have a different coordinate system due to the presence of insertions and deletions (indels). In any of the above queries, the sample $S$ used for the coordinate space can be any of the samples present in the system. If the collection of samples includes a standard reference genome (such as GRCh37), the queries can be presented using that coordinate system. If a known region in a particular sample is of interest, the queries can be presented in that sample's coordinates. Particularly usefully, if the collection contains two "reference" genomes (such as GRCh37 and GHCh38), queries can be posed in either of those coordinate systems. This avoids the effort of calling variants on all the existing samples based on the new reference [23].

Supporting variant queries based on multiple sample coordinate systems requires maintaining a function per sample that can map a position in the reference coordinate (as present in VCF files) to the sample coordinate. Maintaining thousands of such functions requires storing and accessing an order of magnitude more data than only indexing variants based on a single reference coordinate system. Efficiently supporting thousands of coordinate systems adversely affects the memory footprint and computational complexity of the system making this problem much more challenging. This limits the scalability of variant indexes that support multiple coordinate systems to variation data containing only a few thousands samples.

VG toolkit [24] is one of the most widely used tools to represent genomic variation data, and it also supports multiple coordinate systems. It encodes genomic variants from mul- 
tiple samples in a graph, called a variation graph. A variation graph is a sequence graph [25] where each node represents a sequence and a set of nodes through the graph, known as a path, embeds the complete sequence corresponding to each sample. Each node on a path is assigned a position indicating the location of the sequence in the coordinate system of the path. A node can be assigned multiple positions based on the number of paths that pass through the node. The variation graph enables read alignment against multiple sample sequences containing variants simultaneously and avoids mapping biases that arise when mapping reads to a single reference sequence [24, 26-31].

VG toolkit stores each sample path as a list of nodes in the graph and maintains a separate index corresponding to the coordinates of the reference and samples. Storing a separate list of nodes for each sequence impedes the scalability of the representation for storing variation from thousands of samples. Moreover, variants are often shared among samples, so storing a list of nodes for each sample path introduces redundancy in the representation. VG toolkit is designed to optimize read alignment and uses sequence-based indexes for alignment [32,33]. It cannot be directly used for variant queries that require an index based on the position of variants in multiple sequence coordinates. Finally, the VG toolkit representation does not store phasing information contained in VCF files, which is required in many analyses.

Multiple solutions have been proposed that efficiently index variants and support a subset of the variant queries described above. GQT [34] was the first tool that proposed a sample-centric index for storing and querying variants. It stores variants in compressed bitmap indexes and supports efficient comparisons of sample genotypes across many variant positions. BGT [35] and GTC [36] proposed variant-centric indexes that store variants in compressed bit matrices. They support queries for variants in a given region and allow filtering returned variants based on subsets of samples. The SeqArray library [37] is another variant-centric tool for the $\mathrm{R}$ programming language to store and query variants.

However, these tools index variants only in the reference coordinate system and do not support variant queries in a sample coordinate system. Supporting multiple coordinate systems is a much harder problem. Furthermore, these tools do not store the reference sequence and cannot be directly used to query and compare sample sequences in a given region. Other tools have been proposed that use traditional database solutions, such as SQL and NoSQL [38-40]. However, they have proven prohibitively slow to index and query collections of variants.

VariantStore bridges the gap between the tools that are space-efficient and fast but only support reference-based queries (e.g., GTC [36]) and VG, which maintains multiple coordinate systems by storing variants in a variation graph but fails to scale to thousands of samples. We show this by indexing variants from both the 1000 Genomes and TCGA projects ( $>8 \mathrm{~K}$ samples) and show that VariantStore is faster than VG and takes less memory and disk space. VariantStore performs variant queries based on sample coordinates in less than a second. Furthermore, we have designed VariantStore to efficiently scale out of RAM to storage devices in order to cater to the ever increasing sizes of available variation data by performing memory-efficient construction and query.

We encode genomic variation in a directed, acyclic variation graph and build a position index (a mapping of node positions to node identifiers) on the graph to quickly access a node in the graph corresponding to a queried position. Each node in the variation graph 
corresponds to a variant and stores a list of samples that contain the variant along with the position of the variant in the coordinate of those samples. The inverted index design allows one to quickly find all the samples and positions in sample coordinates corresponding to a variant. It also avoids redundancy that otherwise arises in maintaining individual variant indexes for each sample coordinate and scales well in practice when the number of samples grows beyond a few thousand.

To efficiently scale to thousands of coordinate systems (or samples), we maintain the position index only on a single sample coordinate system (often the reference genome in the input $\mathrm{VCF}$ ). The position index maps positions in the sample sequence where there is a variant to nodes corresponding to those variants in the variation graph. The sequence chosen for the position index is called the marker sequence and the nodes on the path of the marker sequence act as marker nodes in the variation graph. To lookup a position using a sample's coordinate system, we first lookup the marker node corresponding to the position in the marker sequence using the position index. We then traverse the sample path from the marker node in the graph to determine the appropriate node in the sample coordinate. A node with a given position in a sample coordinate is often close to the node in the marker sequence coordinate with the same position.

Maintaining the position index only on the marker sequence also makes it easier to include multiple references in the same index. Each new reference can be treated as a sample. Adding a new reference sequence to the index requires adding the variation in the new reference based on the previous reference already in the index. Furthermore, to speed up searches based on the new reference, one can also add a new position index mapping the nodes corresponding to the new reference. We can then use the new position index to directly locate nodes corresponding to positions in the coordinate of the new reference. By enabling queries in any sample's coordinate system, we eliminate the artificial dichotomy between predetermined reference sequences and other samples.

To perform index construction and query efficiently in terms of memory, we partition the variation graph into small chunks (usually a few MBs in size) based on the marker sequence coordinates and store variation graph nodes in these chunks. During construction, only the active chunk is maintained in memory in which new nodes are added, and once it reaches its capacity, we compress and serialize it to disk and create a new active chunk. The nodes in and across chunks are ordered based on the marker sequence coordinate. During a query, we only load the chunks in memory that contain the nodes corresponding to the query range. VariantStore can control the memory usage by tuning the chunk size. This enables VariantStore to scale to very large datasets even on commodity machines where the working memory is often scarce.

\section{Results}

Our evaluation of VariantStore is based on four parameters: construction time, query throughput, disk space, and peak memory usage.

To calibrate our performance numbers, we compare VariantStore against VG toolkit [41]. VG toolkit represents variants in a variation graph and supports multiple coordinate systems similar to VariantStore but does not support variant queries. Therefore, we use the VG toolkit representation as a baseline for space usage and construction time in our evaluation as it stores the same information as the VariantStore. The main difference between VariantStore and the VG toolkit is that VG toolkit uses a separate index corre- 
sponding to each coordinate system whereas VariantStore stores a single index of marker nodes in the variation graph. Comparison with VG toolkit also allows us to evaluate the scalability of existing variation graph indexing tools to index variants from thousands to samples. We do not include VG toolkit in our query evaluation as it does not support the variant queries supported by VariantStore.

\section{Data}

We use 1000 Genomes Phase 3 data [42] and three of the biggest projects from TCGA in terms of the number of samples, Ovarian Cancer (OV), Lung Adenocarcinoma (LUAD), and Breast Invasive Carcinoma (BRCA), for our evaluation. 1000 Genomes data contains more variants compared to the TCGA data but TCGA data contains more samples. 1000 Genomes data contains a separate VCF file for each chromosome containing variants from thousands of samples. The number of samples in each file is $\approx 2.5 \mathrm{~K}$. The variants in 1000 Genomes project are based on GRCh37 reference genome. The TCGA data contains a multiple separate VCF files. The OV, LUAD, and BRCA projects contain 2436, 2680, and 4319 VCF files, respectively. Each VCF file contains two variant types: normal and tumor. For each project in TCGA, we first merged VCF files using the BCF tool merge command [43] and created a separate VCF file for each chromosome. The variants in the TCGA project are based on GRCh38 reference genome.

\section{Index construction}

The total time taken to construct the variation graph representation and index includes the time taken to read and parse variants from the VCF file, construct the variation graph representation and indexes, and serialize the final representation to disk. For VariantStore, the reported time includes the time to create and serialize the position index. The space reported for VariantStore is the sum of the space of the variation graph representation and position index.

For VG toolkit, creating a variation graph representation with multiple coordinate systems (or sample path annotations) requires creating two indexes, XG and GBWT index. The XG index is a succinct representation of the variation graph without path annotations that allows memory- and time-efficient random access operations on large graphs. The GBWT (graph BWT) is a substring index for storing sample paths in the variation graph. We first create the variation graph representation using the "construct" command including all sample path annotations. We then create the XG index and GBWT index from the variation graph representation to create an index with all sample path annotations in the variation graph. For VG toolkit, the reported time includes the time to create and serialize the XG and GBWT indexes. The space reported for VG toolkit is the sum of the space of the XG and GBWT indexes. VG toolkit could not build GBWT index on TCGA data even after running for more than a day. We only report space for the XG index (which does not contain any sample path annotations) for TCGA data.

For both VariantStore and VG toolkit, we created 24 separate indexes, one each for chromosomes 1-22 and X and Y. Each of these indexes were constructed in parallel as a separate process. We report the time taken for construction as the time taken by the process that finished last. Index construction was slowest for chromosome 2 in 1000 Genomes data for both VG toolkit and VariantStore. In TCGA data, chromosome 1 was the slowest. For disk space, we report the total space taken by all 24 indexes. For peak 
memory usage, we report the highest individual and aggregate peak RAM usage for all processes.

The performance of VariantStore and VG toolkit for constructing the index on the 1000 Genomes and TCGA data is shown in Table 1.

VariantStore is $3 \times$ faster, takes $25 \%$ less disk space, and $3 \times$ less peak RAM than VG toolkit. For the TCGA data, VG toolkit could not build GBWT index embedding all sample paths. However, even the space needed for the XG index (not embedding sample paths) is $\approx 3.3 \times$ larger than the VariantStore representation containing all sample paths.

\section{Query throughput}

We measured the query throughput for all four query types mentioned above. To show the robustness of query efficiency on different data, we evaluate on two different chromosome indexes from two different projects. We chose chromosome 2 which is one of the bigger chromosomes and chromosome 22 which is one of the smaller ones. We evaluate query time on chromosome indexes from 1000 Genomes and TCGA LUAD data.

To perform queries, we specify a pair of positions in a sample coordinate system depending on the query type and a sample name. To evaluate the effect of querying based on a sample other than the marker sequence (reference sequence), we perform two types of queries, finding the sequence in a region and finding the variants in a region, using both the marker sequence and a random sample from the input VCF. Specifically, we perform the following six types of queries:

1 ClosestVar $(R ; X)$ : closest variant to $X$ in the marker sequence coordinates

$2 \operatorname{Seq}(R, S ; X, Y)$ : sequence for sample $S$ between $X$ and $Y$ in the marker sequence coordinates

$3 \operatorname{Seq}(S ; X, Y)$ : sequence for sample $S$ between $X$ and $Y$ in its coordinates

$4 \operatorname{Vars}(R, S ; X, Y)$ : variants for sample $S$ between $X$ and $Y$ in the marker sequence coordinates

Table 1 Time, space, peak RAM, and peak RAM (aggregate) to construct variant index on the 1000 Genomes and TCGA (OV, LUAD, and BRCA) data using VariantStore and VG toolkit. *VG toolkit could not build GBWT index embedding all sample paths for TCGA data. Space reported is for the XG index that does not contain any path information. We constructed all 24 chromosomes (1-22 and $X$ and $Y$ ) in parallel. The time and peak RAM reported is for the biggest chromosome (usually chromosome 1 or 2). The space reported is the total space on disk for all 24 chromosomes. The peak RAM (aggregate) is the aggregate peak RAM for all 24 processes

\begin{tabular}{|c|c|c|c|c|}
\hline System & Time & Disk space & Peak RAM & Peak RAM Agg. \\
\hline Dataset & \multicolumn{4}{|c|}{1000 Genomes } \\
\hline VariantStore & $3 \mathrm{~h} 25 \mathrm{~min}$ & $41 \mathrm{~GB}$ & $8.8 \mathrm{~GB}$ & $153 \mathrm{~GB}$ \\
\hline VG toolkit & $11 \mathrm{~h} 10 \mathrm{~min}$ & $50 \mathrm{~GB}$ & $37 \mathrm{~GB}$ & $450 \mathrm{~GB}$ \\
\hline Dataset & \multicolumn{4}{|c|}{ TCGA (OV) } \\
\hline VariantStore & \multirow[t]{2}{*}{$1 \mathrm{~h} 5 \mathrm{~min}$} & $3.4 \mathrm{~GB}$ & \multirow[t]{2}{*}{$1.1 \mathrm{~GB}$} & \multirow[t]{2}{*}{$17.45 \mathrm{~GB}$} \\
\hline VG-toolkit & & 11 GB* & & \\
\hline Dataset & \multicolumn{4}{|c|}{ TCGA (LUAD) } \\
\hline VariantStore & \multirow[t]{2}{*}{$1 \mathrm{~h} 20 \mathrm{~min}$} & $3.5 \mathrm{~GB}$ & \multirow[t]{2}{*}{$2.3 \mathrm{~GB}$} & \multirow[t]{2}{*}{$36.05 \mathrm{~GB}$} \\
\hline VG toolkit & & $12 \mathrm{~GB}^{*}$ & & \\
\hline Dataset & \multicolumn{4}{|c|}{ TCGA (BRCA) } \\
\hline VariantStore & \multirow[t]{2}{*}{$4 \mathrm{~h} 36 \mathrm{~min}$} & $4.2 \mathrm{~GB}$ & \multirow[t]{2}{*}{$3.2 \mathrm{~GB}$} & \multirow[t]{2}{*}{$53.21 \mathrm{~GB}$} \\
\hline VG toolkit & & $14 \mathrm{~GB}^{*}$ & & \\
\hline
\end{tabular}


$5 \operatorname{Vars}(S ; X, Y)$ : variants for sample $S$ between $X$ and $Y$ in its coordinates

$6 \operatorname{AllVars}(R ; X, Y)$ : all variants between $X$ and $Y$ in the marker sequence coordinates

VariantStore supports Closest $\operatorname{Var}(S ; X)$ and $\operatorname{AllVars}(S ; X, Y)$ for any $S$. In our experiments, we present results for $S$ equal to the marker sequence for those queries because they are representative of the performance of those queries.

During a query, we only load the position index and graph topology completely in memory which are only a small portion of the total index space. The nodes in the variation graph representation are partitioned into chunks and only relevant chunks corresponding to the query range are loaded in memory. Keeping the variation graph representation on disk keeps the peak memory usage low and all disk accesses are performed during the query to load appropriate node chunks.

Given a batch of queries, we first sort the queries based on the starting position (treating input positions in the marker coordinate system for all query types) and then perform the queries in order. Performing queries in sorted order ensures that we always access node chunks in sequential order and only have to load a chunk once to answer all queries corresponding to the range of nodes it contains. It also amortizes the cost of loading and unloading node chunks to and from disk.

We performed three sets of query benchmarks containing 10,100, and 1000 queries and report the aggregate time. For each query in the set, we uniformly randomly pick the start position across the full chromosome length. The size of the query range is set to $\approx 42 \mathrm{~K}$ bases which is approximately the length of a typical gene. Picking multiple query regions uniformly randomly across the chromosome provides a good coverage of different regions across the chromosome.

The query throughput on 1000 Genomes data is shown in Figs. 1a and 2a. For most query types, the aggregate time taken to execute queries increases sublinearly with the number of queries. This is because as the batch size increases the cost of loading and unloading node chunks is amortized against higher number of queries. Finding the sequence corresponding to a sample in a region (Seq(RS) and Seq(S)) takes less time compared to finding variants in a region $(\operatorname{Vars}(\mathrm{RS})$, $\operatorname{Vars}(\mathrm{S})$, and AllVars $(\mathrm{R}))$. Finding the sequence takes less time because it involves traversing the sequence specific path in the region and reconstructing the sequence. However, finding variants in a given region takes more time because it involves an exhaustive search of neighbors at each node in the region to determine all the variants that are contained by a given sample or all samples.

The query throughput on TCGA data is shown in Figs. $1 \mathrm{~b}$ and 2b. The TCGA data has twice as many samples compared to 1000 Genomes data but there are fewer variants. This makes the variation graph much sparser and queries in the position index become more expensive compared to traversing the graph between two positions. Finding all variants is the fastest query because the variation graph is very sparse and most position ranges are empty. Traversing the graph to find the sequence or variants for a sample takes similar amount of time. Finding the closest variant from a position takes the most amount of time because it involves performing multiple position index queries to determine the closest variant.

For both 1000 Genomes and TCGA LUAD data, finding the closest variant query is faster for chromosome 2 because variants in chromosome 22 are more dense compared to chromosome 2 which makes it faster to locate the closest variant. 


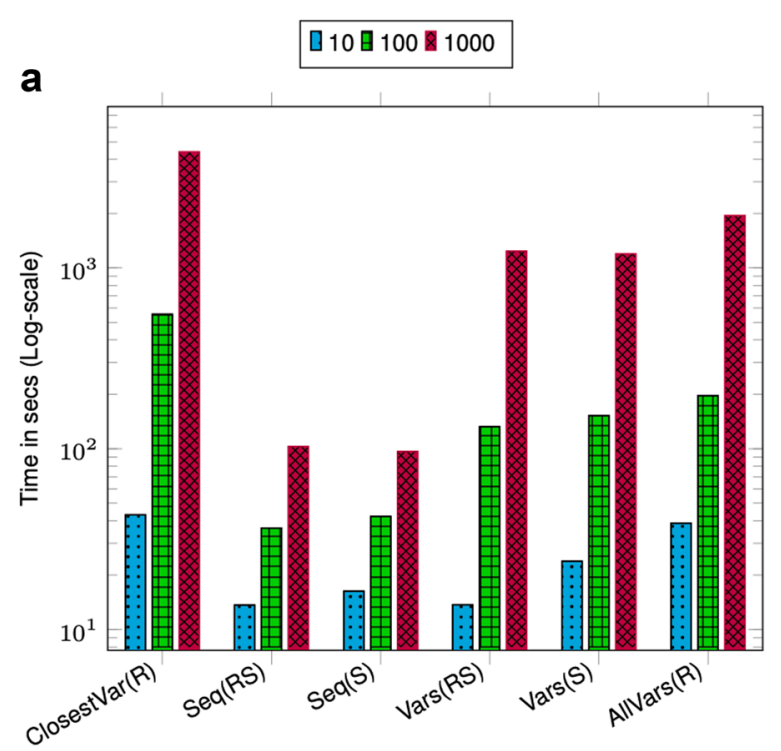

b

(a) 1000 Genomes data

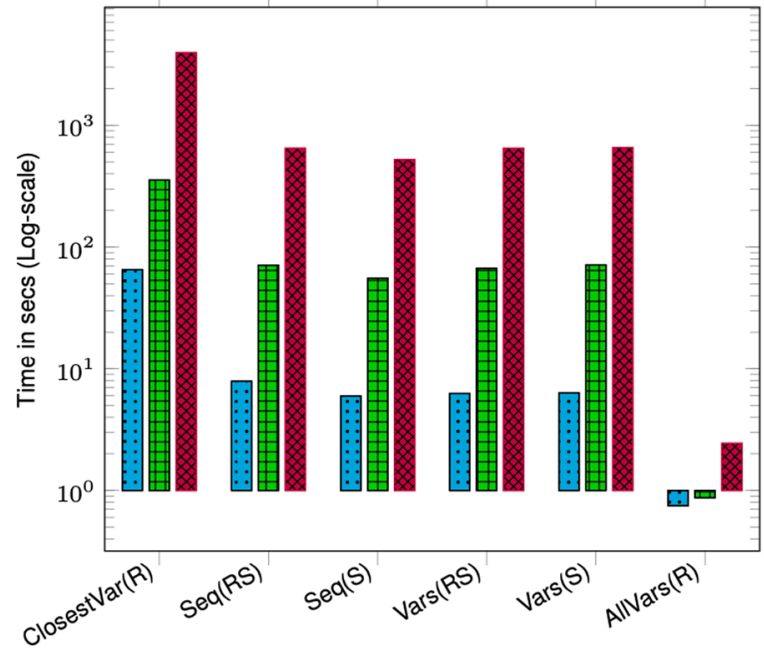

(b) TCGA LUAD data

Fig. 1 Chromosome 22 query performance. Time reported is the total time taken to execute 10, 100, and 1000 queries. For all queries, the query length is fixed to $\approx 42 K$. ClosestVar(R), Closest variant; Seq(RS), Seq in ref coordinate; Seq(S), Seq in Sample coordinate; Vars(RS), Sample variants in ref coordinate; Vars(S), Sample variants in Sample coordinate; AllVars(R), All variants in ref coordinate

The effect of querying based on a sample other than the marker sequence is negligible as shown in the timing results for Seq(RS) and Seq(S) and query $\operatorname{Vars}(\mathrm{RS})$ and $\operatorname{Vars}(\mathrm{S})$. This shows that the overhead of the local graph search to convert the position from the coordinate of the marker sequence to the sample sequence is low.

\section{The effect of query range on peak memory usage}

We performed another query benchmark to evaluate the effect of size of the position range on the peak memory usage and time. For this benchmark, we chose the "Sample sequence in marker coordinate" query (Seq(RS)) because this query involves traversing the full sample path between two positions. We performed sets of 100 queries with 

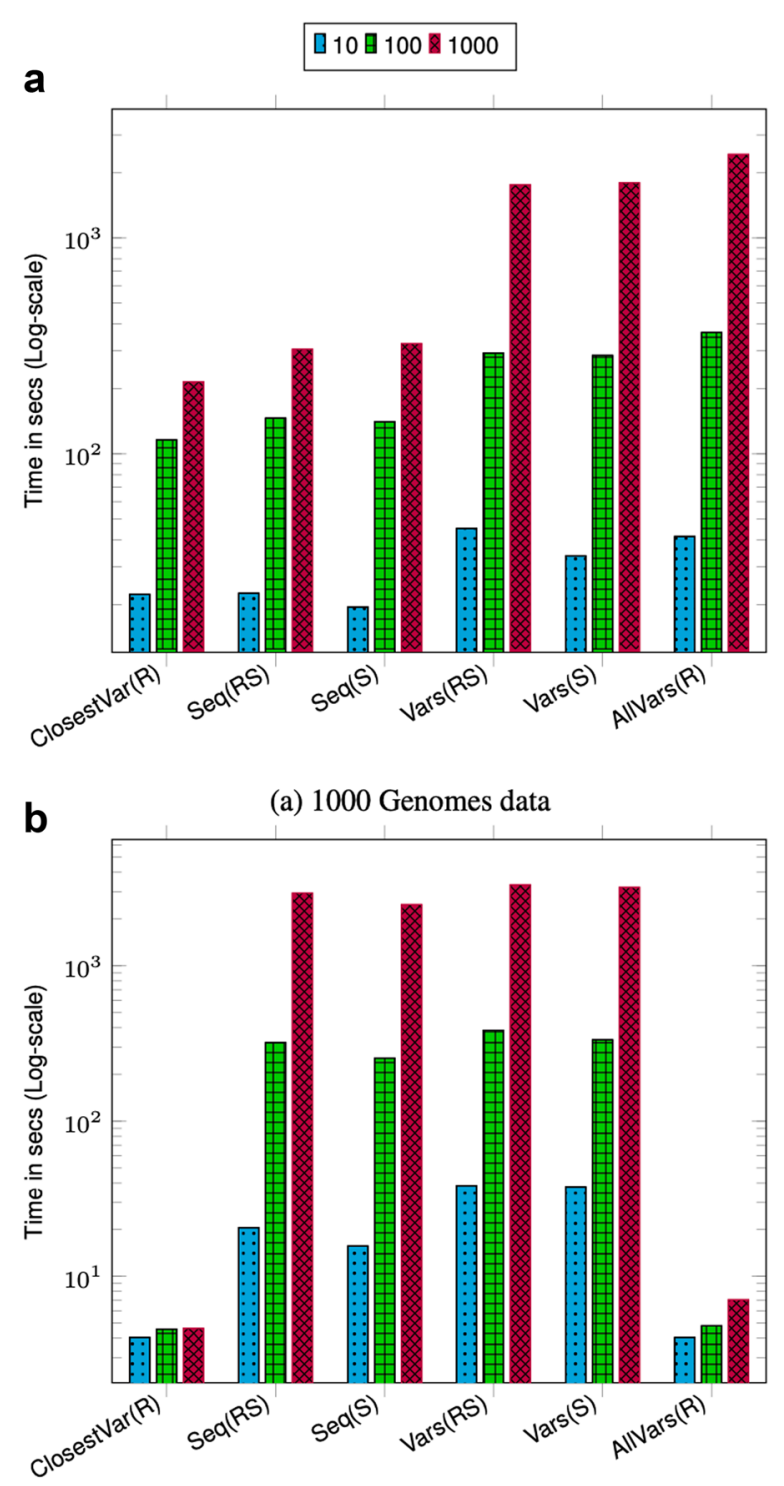

(b) TCGA LUAD data

Fig. 2 Chromosome 2 query performance. Time reported is the total time taken to execute 10, 100, and 1000 queries. For all queries the query length is fixed to $\approx 42 \mathrm{~K}$. Closest $\operatorname{Var}(\mathrm{R})$, Closest variant; Seq(RS), Seq in ref coordinate; Seq(S), Seq in Sample coordinate; Vars(RS), Sample variants in ref coordinate; Vars(S), Sample variants in Sample coordinate; AllVars(R), All variants in ref coordinate

increasing size of the position range and record the total time and peak RAM usage. For each query in the set, we uniformly randomly pick the start position across the full chromosome length.

The effect of the query range size on peak memory usage and time is shown in Fig. 3. The memory usage remains constant regardless of the query length. This is because during a query we access node chunks in sequential order and regardless of the query length only load at most two node chunks in RAM at a time. This keeps the memory usage essentially constant. 


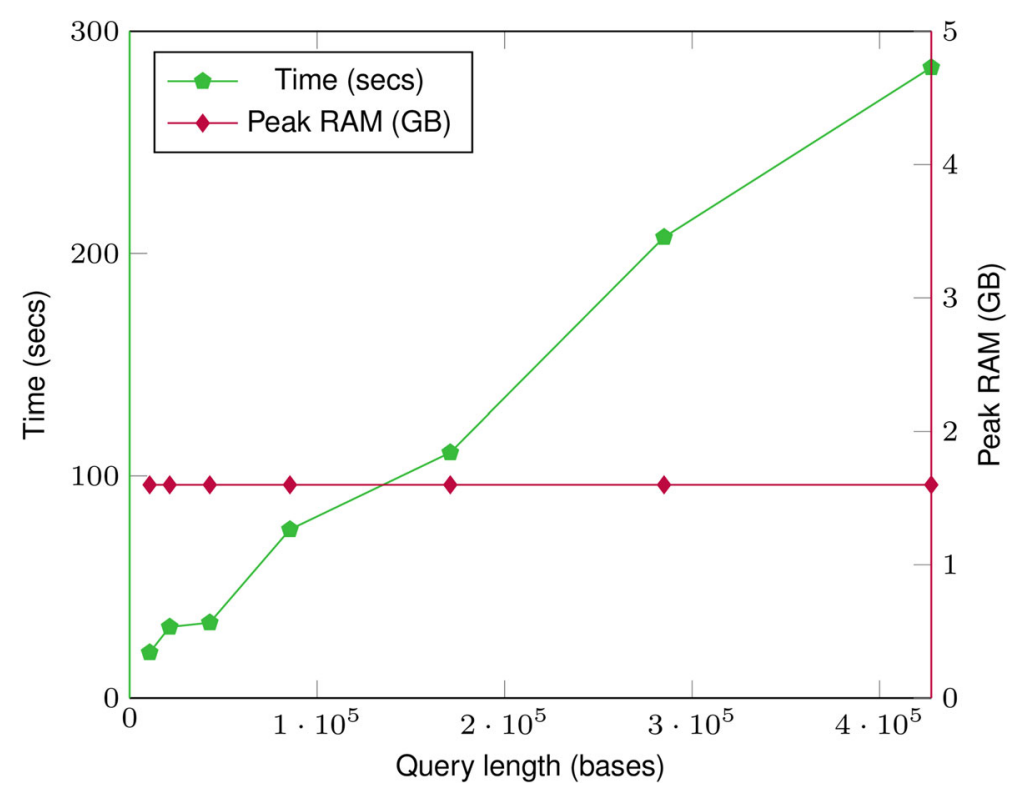

Fig. 3 Time (seconds) and peak memory usage (GB) with increasing query length for "Sample sequence in the marker coordinate" (Seq(RS)) for 1000 Genomes chromosome 22 index. The time taken increases as the query length increases. But the memory usage remains constant regardless of the query length

The peak memory usage depends on the size of node chunks and is not a hard constraint. It can be tuned to a lower or higher value depending on the system requirements by changing the size of node chunks.

\section{The effect of number of variants on query time}

We also evaluate how the number of variants in the position range affects the query time. For this benchmark, we performed the "All variants in the marker coordinate" query (All$\operatorname{Vars}(\mathrm{R})$ ) because this query involves performing a breadth-first search in the graph to determine all variants in a region and the query time depends on the number of variants in the region. To perform queries on regions with different number of variants, we chose 1000 regions with a fixed size of the position range $(\approx 42 \mathrm{~K}$ bases $)$ and start position chosen uniformly randomly across the chromosome.

The effect of the number of variants in the query region on query time is shown in Fig. 4. The query time increases as the number of variants in the queried region increases. This is because when the number of variants in a region is small the graph is sparser and faster to traverse and report all variants.

\section{VariantStore index space analysis}

Figure 5 shows the distribution of space taken by different components in VariantStore. There are four major components and distribution of space varies depending on data being indexed. The average number of samples that share a variant is much higher in 1000 Genomes data compared to TCGA data. In VariantStore, we store information about each sample that shares the variant in the node corresponding to the variant. This causes more information being stored in each node and makes the node list the dominant component of the index in 1000 Genomes index. However, TCGA data is much sparser in terms of 


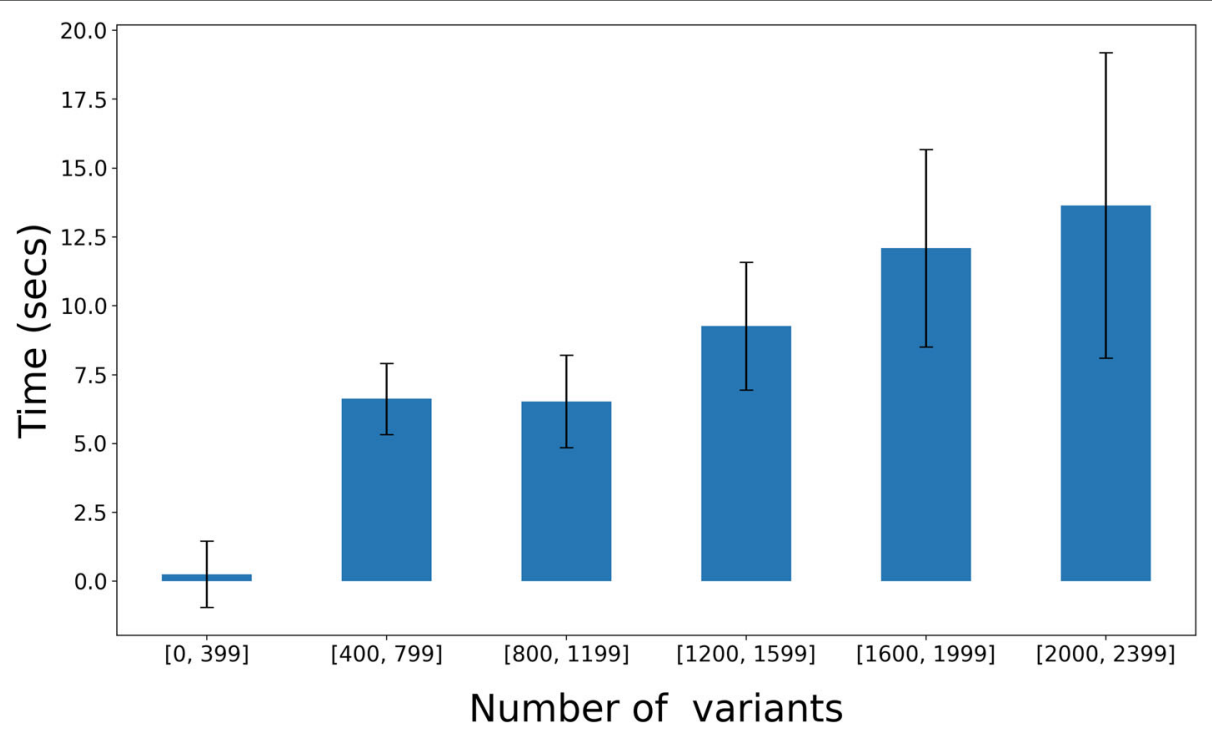

Fig. 4 Time (seconds) and the number of variants in the query region for "All variants in the marker coordinates" (AllVars(R)) for 1000 Genomes chromosome 22 index. Query times are binned based on the number of variants in the query region and mean time is reported in each bin. Error bars are standard deviation. The mean time increases as the number of variants increases in the region

the number of samples sharing a variant. Therefore, each node stores less information and the graph topology becomes the major component.

Across all indexes in Fig. 5, the position index only forms a small percentage (1.5-4.5\%) of the overall index. This is because we maintain the position index only for the nodes corresponding to the marker sequence. The small size of the position index allows us to add multiple reference sequences in the same VariantStore index with only a small space overhead.

\section{Comparison with a reference-based variant index}

To understand the overhead of maintaining thousands of coordinate systems on the performance of VariantStore, we compare VariantStore to a variant index that only indexes variants based on the reference coordinate system and supports a subset of variant queries. We use GTC [44] in our evaluation as it is the fastest and smallest reference-based variant index. We compare the query performance for two variant queries (Vars(RS) "Sample variants in ref coordinate" and AllVars(R) "All variants in ref coordinate") supported by GTC.

GTC took $6 \times$ less time and an order of magnitude less space to construct and store the variant index compared to VariantStore. Furthermore, variant queries were also about an order of magnitude faster in GTC (see Fig. 6). The slow performance of VariantStore is due to the overhead of maintaining the variation graph for representing multiple coordinate systems. During index creation, adding a variant requires splitting a marker node, adding the variant node in the graph, and updating the mapping function for all the samples corresponding to the variant. To query, we first map the variant position to a node in the graph using the position index and then traverse the path in the graph to answer queries. On the other hand, adding and querying variants can be performed fairly efficiently using compressed bit vectors in reference-only variant indexes. If an application 
:- Position Index $⿴$ Graph topology Node list $\square$ Sequence buffer

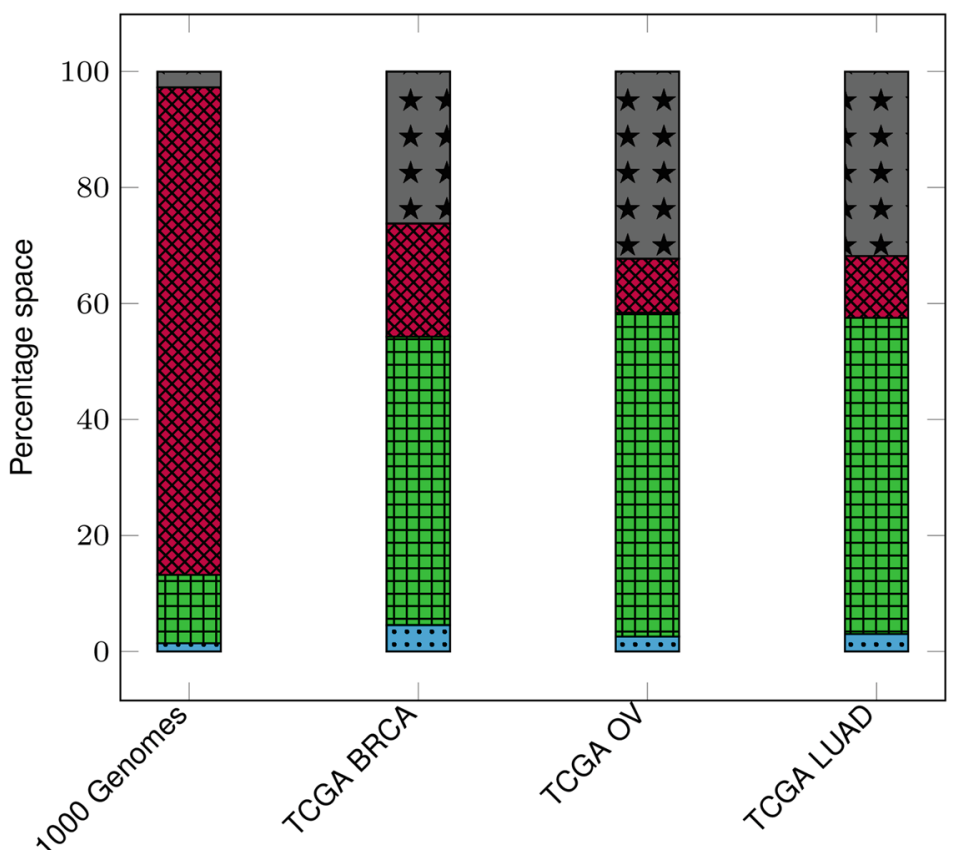

Fig. 5 Percentage distribution of space requirements of different components in VariantStore for 1000 Genomes and TCGA data

only requires querying variants based on a single reference coordinate system, then GTC offers a space-efficient and faster alternative, but it does not support queries using persample coordinate systems or general genome graph traversals. Representing variation data as a genome graph also has usage in various other applications [24, 26-31, 45-48].

\section{VariantStore index with multiple standard references}

To show the usability of VariantStore to analyze genomic variants across multiple versions of standard references, we constructed a VariantStore index that included two versions of the human reference genome: GRCh37 and GRCh38. A genomic variant index with multiple references enables us to quickly analyze genomic variants across different versions of these standard references without having to perform the costly task of variant calling for individual samples based on the new reference version.

To do this, we treat the second reference genome (GRCh38) as another sample and insert variants from GRCh38 based on GRCh37. In addition to these two standard references, we include variants from the 1000 Genomes project (which are based on GRCh37). We only constructed the position index on the GRCh37 sequence.

We called variants for human reference version GRCh38 based on version GRCh37 using Minimap2 [49] and converted the output from Minimap2 to a VCF file using Samtools [50] and freebayes [51]. We merged the VCF file containing variants of reference version GRCh38 with the variants from 1000 Genomes project which are also based on the reference version GRCh37 using BCFtools [43]. We then constructed an index using the merged VCF file. 


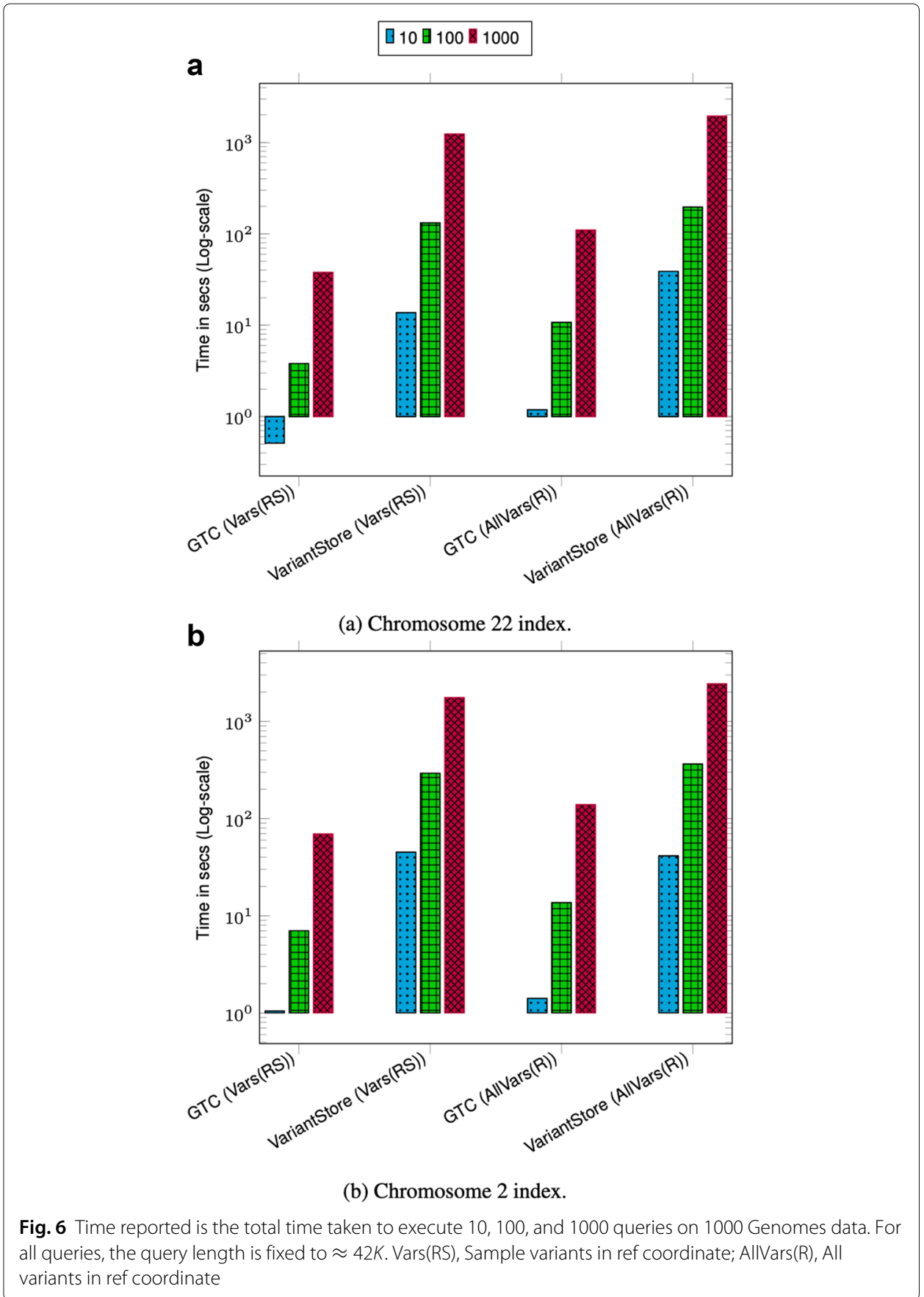

Table 2 shows the construction performance of the VariantStore with and without the reference GRCh38. Adding the variants from GRCh38 to the variants from the 1000 Genomes projects only increases the construction time by $15 \%$ and peak memory by $4 \%$ and has a negligible effect on the index size. Figure $7 \mathrm{a}$ and $\mathrm{b}$ show the query performance based on the two versions of the reference genome. Querying for variants based on the GRCh38 is slightly slower as the position index is only built for the GRCh37 sequence. We can use the position index to directly locate nodes in the GRCh37 coordinate but locating nodes in the GRCh38 requires local graph search. 
Table 2 Time, space, peak RAM, and peak RAM (aggregate) to construct variant index on the 1000 Genomes data with and without GRCh38 as an another sample. We constructed all 24 chromosomes (1-22 and $X$ and $Y$ ) in parallel. The time and peak RAM reported is for the biggest chromosome (usually chromosome 1 or 2). The space reported is the total space on disk for all 24 chromosomes. The peak RAM (aggregate) is the aggregate peak RAM for all 24 processes

\begin{tabular}{lllll}
\hline System & Time & Disk space & Peak RAM & Peak RAM Agg. \\
\hline VariantStore & 3 h $25 \mathrm{~min}$ & $41 \mathrm{~GB}$ & $8.8 \mathrm{~GB}$ & $153 \mathrm{~GB}$ \\
VariantStore (with GRCh38) & 3 h $57 \mathrm{~min}$ & $41 \mathrm{~GB}$ & $9.17 \mathrm{~GB}$ & $179 \mathrm{~GB}$ \\
\hline
\end{tabular}

\section{Experimental hardware}

All experiments on 1000 Genomes data were performed on an Intel Xeon CPU E52699A v4 @ 2.40GHz (44 cores and 56MB L3 cache) with 1TB RAM and a 7.3TB HGST HDN728080AL HDD running Ubuntu 18.04.2 LTS (Linux kernel 4.15.0-46-generic) and were run using a single thread. Benchmarks for TCGA data were performed on a cluster machine running AMD Opteron Processor 6220 @ 3GHz with 6MB L3 cache. TCGA data was stored on a remote disk and accessed via NFS.

\section{Conclusions}

We attribute the scalability and efficient index construction and query performance of VariantStore to the variant-oriented index design. VariantStore uses an inverted index from variants to the samples which scales efficiently when multiple samples share a variant which is often seen in genomic variation data. The inverted index design further allows us to build the position index only on the marker sequence and use graph traversal to transform the position in marker sequence coordinates to sample coordinates.

All the supported variant queries look for variants in a contiguous region of the chromosome which allows VariantStore to partition the variation graph representation into small chunks based on the position of nodes in the chromosome and sequentially load only the relevant chunks into memory during a query. This makes VariantStore memoryefficient and scale to genomic variation data from hundreds of thousands of samples in future.

The variation graph representation in VariantStore is smaller and more efficient to construct than the representation in VG toolkit. It can be further used in read alignment as a replacement to the variation graph representation in the VG toolkit.

While the current implementation does not support adding new variants or updating the marker sequence in an existing VariantStore, this is not a fundamental limitation of the design. In future, we plan to extend the immutable version of VariantStore to support dynamic updates following the LSM-tree design [52].

\section{Methods}

Variation graph

A variation graph (VG) [24] (also defined as a genome graph [29, 30]) is a directed, acyclic graph (DAG) $G=(N, E, P)$ that embeds a set of DNA sequences. It comprises a set of nodes $N$, a set of direct edges $E$, and a set of paths $P$. For DNA sequences, we use the alphabet $\{\mathrm{A}, \mathrm{C}, \mathrm{G}, \mathrm{T}, \mathrm{N}\}$. Each $n_{i} \in N$ represents a sequence $\operatorname{seq}\left(n_{i}\right)$. Edges in the graph connect nodes that are followed on a path. Each sample in the VCF file follows a path through the variation graph. The embedded sequence given by the path is the sample 


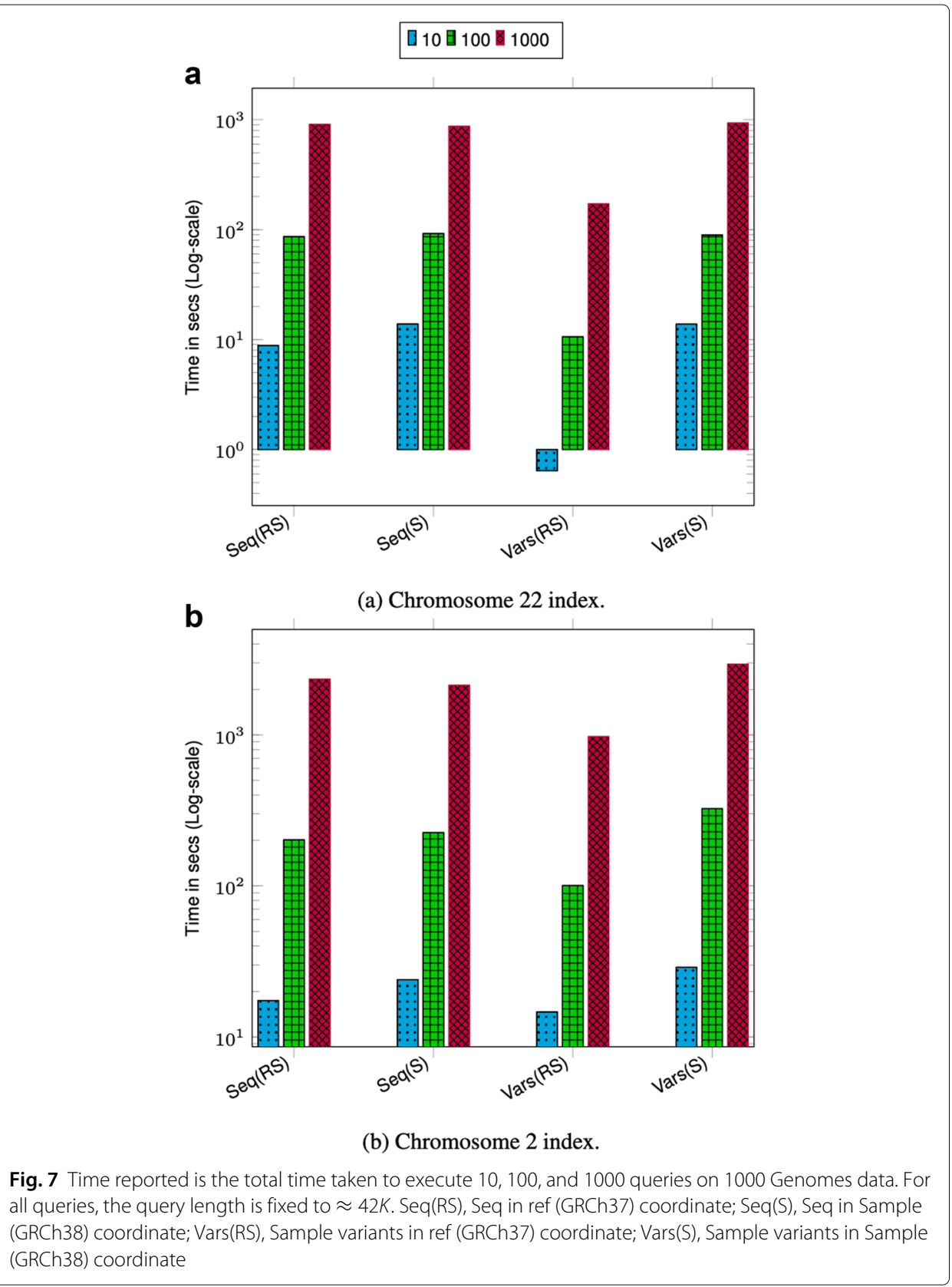

sequence. Given that a variation graph is a directed graph, edges are traversed in only one direction. Although not applicable to VariantStore, an edge can also be traversed in the reverse direction when the variation graph is used for read alignment [24, 29, 30].

Each path $p=n_{s} n_{1} \ldots n_{p} n_{d}$ in the graph is an embedded sequence defined as a sequence of nodes between a source node $n_{s}$ and a destination node $n_{d}$. Nodes on a path are assigned positions based on the coordinate systems of sequences they represent [22]. The position of a node on a path is the sum of the lengths of the sequences represented by nodes traversed to get to the node on the path. For a path $p=n_{1} \ldots n_{p}$, position $P\left(n_{p}\right)$ is $\sum_{i=1}^{p-1}\left|\operatorname{seq}\left(n_{i}\right)\right|$. A node in the graph can appear in multiple paths and therefore can have multiple positions based on different coordinate systems. 
An initial variation graph is constructed with a single node and no edges using a linear marker sequence and a marker genome coordinate system (usually the standard reference genome). Variants are added to the variation graph from one or more VCF files [13]. A variant is encoded by a node in the variation graph that represents the variant sequence and is connected to nodes representing the marker sequence via directed edges. Each variant in the VCF file splits an existing marker sequence node into two (or three in some cases) and joins them via an alternative path corresponding to the variant. For example, a substitution or deletion can cause an existing marker node to be split into three parts (Fig. 8). An insertion can cause the marker node to be split into two parts (Fig. 8).

\section{Representing multiple coordinate systems in a variation graph}

Representing multiple coordinate systems in a variation graph poses challenges that are not present in linear reference genomes. First, a node can appear on multiple paths at a

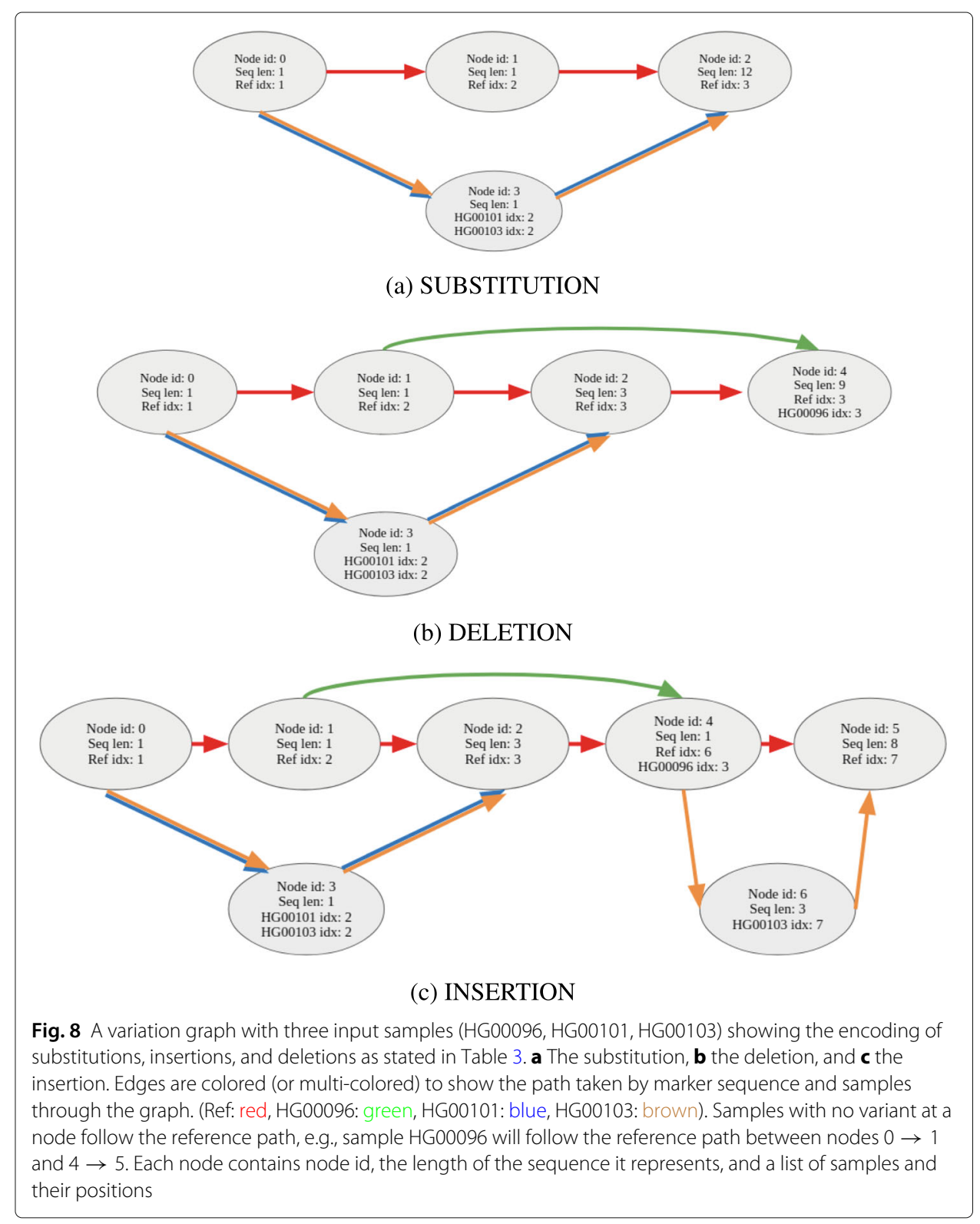


Table 3 Variants ordered by the position in the marker genome for three samples (HG00096, HG00101, HG00103). Each variant has the list of samples that contain the variant

\begin{tabular}{lllllll}
\hline Reference sequence & \multicolumn{6}{l}{ CAATTTGCTGATCT } \\
\hline Position & Reference seq. & Alternative seq. & HG00096 & HG00101 & HG00103 & Variant type \\
2 & A & G & 0 & 1 & 1 & SUBSTITUTION \\
2 & AATT & A & 1 & 0 & 0 & DELETION \\
6 & T & TACG & 0 & 0 & 1 & INSERTION \\
\hline
\end{tabular}

different position on each path. Second, given that a variation graph can contain thousands of paths and coordinate systems, it would be non-trivial to maintain a position index to quickly get to a node corresponding to a path and position.

Much work has been done devising efficient approaches to handle multiple coordinate systems. The offset-based coordinate system was introduced by Rand et al. [22]. VG toolkit [41] implemented the multiple coordinate system by explicitly storing a list of node identifiers and node offsets for each path in the variation graph. They store the list of node identifiers as integer vectors using the succinct data structure library (SDSL [53]). We call this an explicit-path representation.

However, storing the list of nodes on each path explicitly can become a bottleneck as the number of input paths increases. Moreover, nodes that appear on multiple paths are stored multiple times causing redundancy in storage. For a set of $N$ variants and $S$ samples, the space required to store the explicit-path representation is $O(S N)$ since each variant creates a constant number of new nodes in the variation graph.

\section{VariantStore}

We describe how we represent a variation graph in VariantStore and maintain multiple coordinate systems efficiently. We then describe how we build a position index using succinct data structures.

The variation graph representation is divided into three components:

1 Variation graph topology

2 Sequence buffer

3 List of variation graph nodes

\section{Variation graph topology}

A variation graph constructed by inserting variants from VCF files often shows high sparsity (the number of edges is close to the number of nodes). For example, the ratio of the number of edges to nodes in the variation graph on 1000 Genomes data [42] is close to 1 . Given the sparsity of the graph, we store the topology of the variation graph in a representation optimized for sparse graphs.

Our graph representation uses the counting quotient filter (CQF) [54] as the underlying container for storing nodes and their outgoing neighbors. The CQF is a compact representation of a multiset $S$. It associates each key with its count. The CQF supports inserting a key with an arbitrary count. A query for a key $x$ returns the number of instances of $x$ in $S$. The CQF uses a variable-length encoding scheme to efficiently count the multiplicity of keys.

We use the exact version of the counting quotient filter (i.e., no false-positives) to map a node to its outgoing edge(s). We store the node id as the key in the CQF and if there is only one outgoing edge we encode the outgoing neighbor id as the count of the key. If there is more than one outgoing edge, we use indirection. We maintain a list of vectors where each 
vector contains a list of outgoing neighbor identifiers corresponding to a node. We store the node id as the key and index of the vector containing the list of outgoing neighbor id as the count of the key. In the variation graph, most nodes have a single outgoing neighbor and by using the CQF-based compact and efficient graph representation, we achieve fast traversal of the graph.

\section{Sequence buffer}

The sequence buffer contains the marker sequence and all variant sequences corresponding to each substitution and insertion variant. All sequences are encoded using 3-bit characters in an integer vector from SDSL library $[53,55]$. We use the 3-bit encoding as there are five possible values $(\mathrm{A}, \mathrm{C}, \mathrm{G}, \mathrm{T}, \mathrm{N})$ in genomic sequences. The integer vector initially only contains the marker sequence. Sequences from incoming variants are appended to the integer vector. Once all variants are inserted, the integer vector is bit compressed before being written to disk.

\section{List of variation graph nodes}

Each node in the variation graph contains an offset and length. The offset points to the start of the sequence in the sequence buffer, and length is the number of nucleotides in the sequence starting from the offset. This uniquely identifies a node sequence in the sequence buffer.

At each node, we also store a list of sample identifiers that have the variant, position of the node on all those sample paths, and phasing information from the VCF file corresponding to each sample.

Our representation of the list of samples is based on two observations. First, multiple samples share a variant and storing a list of sample identifiers for each variant is space inefficient. Instead, we store a bit vector of length equal to the number of samples and set bits corresponding to the present samples in the bit vector. Second, multiple variants share the same set of samples. We define an equivalence relation $\sim$ over the set of variants. Let $E(v)$ denote the function that maps each variant to the set of samples that have the variant. We say that two variants are equivalent (i.e., $v_{1} \sim v_{2}$ ) if and only if $E\left(v_{1}\right)=E\left(v_{2}\right)$. We refer to the set of samples shared by variants as the sample class. A unique id is assigned to each sample class and nodes store the sample class id instead of the whole sample class. This scheme has been employed previously by colored de Bruijn graph representation tools [56-59] for efficiently maintaining a mapping from $k$-mers (a $k$-length substring sequence) to the set of samples where $k$-mers appear.

Phasing information is encoded using 3 bits: 1 bit to store whether the variant is phased or unphased and 2 bits for the ploidy. Position and phasing information corresponding to each sample in the list of samples is stored as tuples. Tuples are stored in the same order as the samples appear in the sample class bit vector. To retrieve the tuple corresponding to a sample, a rank operation is performed on the sample bit vector to determine the rank of the sample. Using the rank output, a select operation is performed on the tuple list to determine the tuple corresponding to a sample.

Variation graph nodes are stored as protocol buffer objects using Google's open-source protocol buffers library. Every time a new node is created, we instantiate a new protocol buffer object in memory. We compress the protocol buffers before writing them to disk and decompress them while reading them back in memory. 
For a set of $N$ variants and $S$ samples where each variant is shared by $M$ samples on average, each node contains information about $M$ samples and storing $O(N)$ nodes (a constant number of nodes for each variant) the space required to store the variation graph representation in VariantStore is $O(N M)$. When $M=1$ (i.e., no two samples share a variant), the space required by the variation graph representation becomes $O(N)$.

\section{Position index}

In order to answer variant queries, we need an index to quickly locate nodes in the graph corresponding to input positions. These positions can be specified in the coordinate system of any sample.

One way to index the variation graph is to store an ordered mapping from position to node identifier. We can perform a binary search in the map to find the position closest to the queried position and the corresponding node id in the graph. However, given that there are multiple coordinate systems in the variation graph, we cannot create a single mapping with a global ordering. Keeping a separate position index for each coordinate system will require space equal to the explicit-path representation.

In VariantStore, we maintain a mapping of positions to node identifiers only for the marker coordinate system. All nodes on the marker sequence path are present in the mapping. If the queried position is in the marker coordinate system, we use the mapping to locate the node corresponding to the position in the graph. However, if the queried position is in a sample's coordinate system other than the marker, we first locate the node in the graph corresponding to the same position in the marker coordinate system. Although the same position might be shifted in the marker coordinate system, it will point us to the marker node in the graph that is close to the sample node. From the corresponding marker node, we perform a local search by traversing the sample path to determine the node corresponding to the position in sample's coordinate system. The local graph search incurs a small cost because sample nodes are rarely far from a marker node and is amortized against future searches in the sample's coordinate system.

We create the position index using a bit vector called the position-bv of length equal to the marker sequence length and a list of node identifiers on the marker path in the increasing order by their marker sequence positions. For every node in the list, we set the bit corresponding to the node's position in the position-bv. There is a one-to-one correspondence between every set bit in the position-bv and node positions in the list. We store the position-bv using a bit vector and node list as an integer vector from the SDSL library $[53,55]$.

\section{Variation graph construction}

We construct the variation graph by inserting variants from a VCF file. Each variant has a position in the reference genome, alternative sequence (except in case of a deletion), and a list of samples with phasing information for each sample.

Based on the position of the variant, we split an existing marker node that contains the sequence at that position in the graph. We update the split nodes on the marker path with new sequence buffer offsets, lengths, and node positions (based on the marker coordinate system). We then append the alternative sequence to the sequence buffer and create an alternative node with the offset and length of the alternative sequence. We then add the list of tuples (position, phasing info) for each sample. 
We also need to determine the position of the alternative node on the path of each sample that contains the variant. One way to determine the position of the node for each sample would be to backtrack in the graph to determine a previous node that contains a sample variant and the absolute position of that node in the sample's coordinate system. If no node is found with a sample variant, we trace all the way back to the source of the graph. We would then traverse the sample path forward up to the new alternative node and compute the position. This backtracking process would need to be performed once for each sample that contains the variant. This would slow down adding a new variant and cause the construction process to not scale well with increasing number of samples.

Instead, we construct the variation graph in two phases to avoid the backtracking process. In the first phase, while adding variants, we do not update the position of nodes on sample paths. We only maintain the position of nodes on the marker path because that does not require backtracking. In the second phase, we perform a breadth-first traversal of the variation graph starting from the source node and update the position of nodes on sample paths.

During the breadth-first traversal, we maintain a delta value for each sample in the VCF file. At any node, the delta value is the difference between the position of the node in the marker coordinate and the sample's coordinate. During the traversal, we update sample positions for each node based on the current delta value and marker coordinate value. Algorithm 1 gives the pseudocode of the algorithm.

Algorithm 1 Pseudocode to fix sample positions in the variation graph. A node corresponding to a variant contains the list of sample identifiers that have the variant and their respective positions in sample paths. A node corresponding to the marker sequence contains the position in the marker path and optionally a list of sample identifiers if it also represents a delete variant.

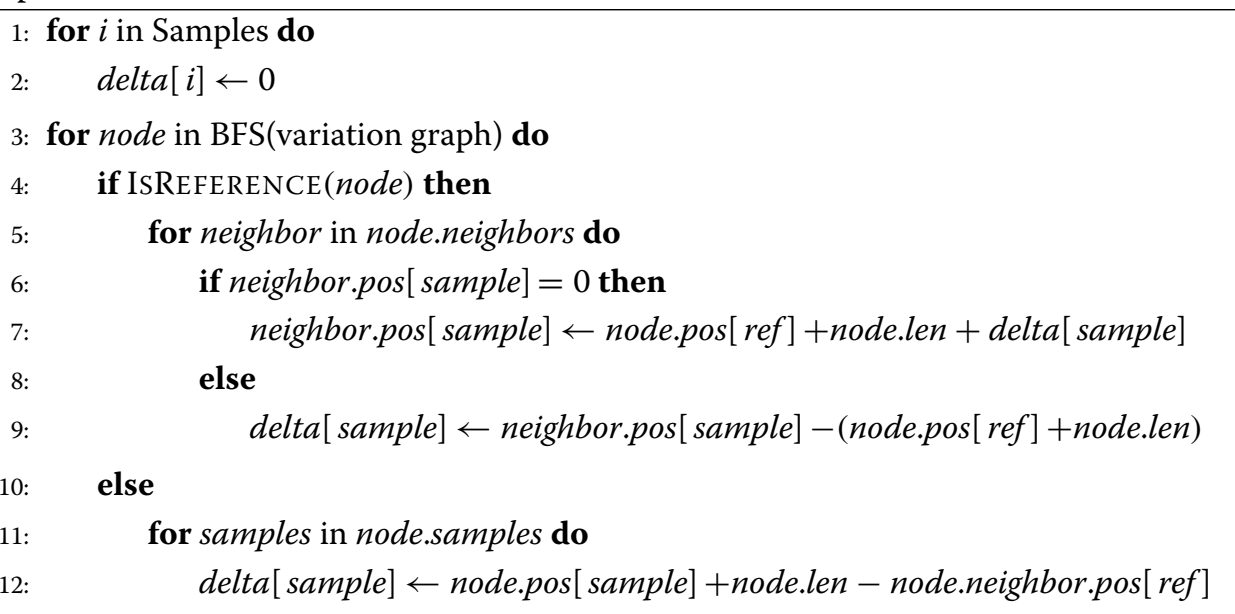

\section{Position index construction}

In the position index, we maintain a mapping from positions of nodes on the marker path to corresponding node identifiers in the graph. Node positions are stored in a "positionbv" bit vector of size equal to the length of the marker sequence and node identifies are stored in a list. To construct the position index, we follow the marker path starting from 
the source node in the graph and for every node on the path we set the corresponding position bit in the position-bv and add the node identifier to the list. Node identifiers are stored in the order of their position on the marker path.

\section{Variant queries}

A query is performed in two steps. We first perform a predecessor search (largest item smaller than or equal to the queried item) using the queried position in the position index to locate the node $n_{p}$ with the highest position smaller than or equal to the queried position pos. The predecessor search is implemented using the rank operation on position-bv. For bit vector $B[0, \ldots, n], R A N K(j)$ returns the number of 1 s in prefix $B[0, \ldots, j]$ of $B$. An RRR compressed position-bv supports rank operation in constant time [53, 60]. The rank of pos in position-bv corresponds to the index of the node id in the node list. Figure 9a shows a sample query in the position index.

Based on how marker nodes are split while adding variants, the sequence starting at pos will be contained in the node $n_{p}$. All queries are then answered by traversing the graph either by following a specific path or a breadth-first traversal and filtering nodes based on query options.

If the queried position is based on the marker coordinate system, then we can directly use $n_{p}$ as the start node for graph traversal. However, if the position is based on a sample coordinate, then we perform a local search in the graph starting from $n_{p}$ to determine the start node based on the sample coordinate.

\section{Memory-efficient construction and query}

In the variation graph representation, the biggest component in terms of space is the list of variation graph nodes stored as Google protobuf objects. These node objects contain the sequence information and the list of sample positions and phasing information. For 1000 Genomes data, the space required for variation graph nodes is $\approx 87$ to $92 \%$ of the total space in VariantStore. However, keeping the full list of node objects in memory during construction or query is not necessary and would make these processes memory inefficient.

To perform memory efficient construction and query, we store and serialize these nodes in small chunks usually containing $\approx 200 \mathrm{~K}$ nodes (the number of nodes in a chunk varies based on the data to keep the size to a few MBs). Nodes in and across these chunks are kept in their creation order (which is roughly the breadth-first traversal order). Therefore, during a breadth-first traversal of the graph, we only need to load these chunk in sequential order.

During construction, we only keep two chunks in memory, the current active chunk and the previous one. All chunks before the previous chunk are written to disk. In the second phase of the construction when we update sample positions and during the position index creation, we perform a breadth-first traversal on the graph and load chunks in sequential order.

Variant queries involve traversing a path in the graph between a start and an end position or exploring the graph locally around a start position. All these queries require bounded exploration of the graph for which we only need to look into one or a few chunks.

To perform queries with a constant memory, we only load the position index and variation graph topology in memory and keep the node chunks on disk. We use the index and 

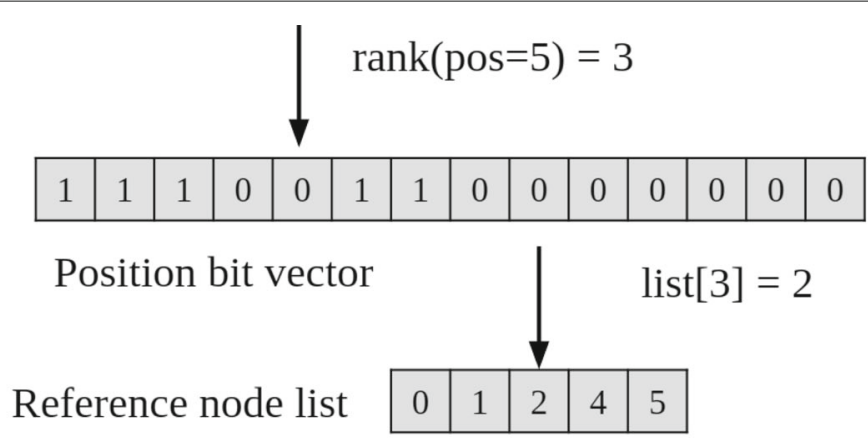

(a) Position Index

\begin{tabular}{|l|l|l|l|l|l|l|l|l|l|l|l|l|l|l|l|l|l|}
\hline & 1 & 2 & 3 & 4 & 5 & 6 & 7 & 8 & 9 & 10 & 11 & 12 & 13 & 14 & 15 & 16 & 17 \\
$\mathrm{C}$ & $\mathrm{A}$ & $\mathrm{A}$ & $\mathrm{T}$ & $\mathrm{T}$ & $\mathrm{T}$ & $\mathrm{G}$ & $\mathrm{C}$ & $\mathrm{T}$ & $\mathrm{G}$ & $\mathrm{A}$ & $\mathrm{T}$ & $\mathrm{C}$ & $\mathrm{T}$ & $\mathrm{G}$ & $\mathrm{A}$ & $\mathrm{C}$ & $\mathrm{G}$ \\
\hline
\end{tabular}

Sequence buffer

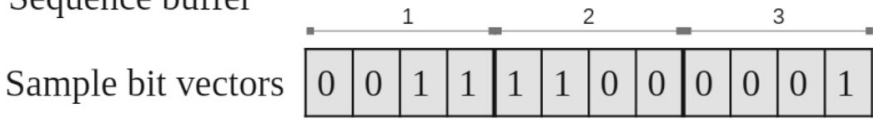

Node list

\begin{tabular}{|c|c|c|c|c|}
\hline ID & Offset & Len & Index & Class Id \\
\hline 0 & 0 & 1 & 1 & 0 \\
\hline 1 & 1 & 1 & 2 & 0 \\
\hline 2 & 2 & 3 & 3 & 0 \\
\hline 3 & 14 & 1 & 2,2 & 1 \\
\hline 4 & 5 & 1 & 6,3 & 2 \\
\hline 5 & 6 & 8 & 7 & 0 \\
\hline 6 & 15 & 3 & 7 & 3 \\
\hline
\end{tabular}

Graph topology

(b) Variation graph representation

Fig. 9 Position index and variation graph representation in VariantStore for the sample graph from Fig. 8. a The query operation for finding the marker node identifier corresponding to position 5 in the marker sequence. The rank operation counts the number of marker nodes on or before position 5 . The result of rank operation is the index in the node list that contains the identifier of the node corresponding to the sequence at position 5. b Phasing information is omitted from the node list for simplicity of the figure. In implementation, phasing information is stored using three bits for each sample in each node

the graph topology to determine the set of nodes to look at to answer the query. We then load appropriate chunks from disk which contain the start and end nodes in the query range. For queries involving local exploration of the graph, we load the chunk containing the start node. During the exploration, we load new chunks lazily as needed. At any time during the query, we only maintain two contiguous chunks in memory.

\section{Supplementary Information}

The online version contains supplementary material available at https://doi.org/10.1186/s13059-021-02442-8. 
Acknowledgements

We would like to thank Shawn Baker for many helpful discussions and Guillaume Marçais and Yutong Qiu for comments on the manuscript.

\section{Peer review information}

Anahita Bishop was the primary editor of this article and managed its editorial process and peer review in collaboration with the rest of the editorial team.

\section{Review history}

The review history is available as Additional file 1.

\section{Authors' contributions}

Conceptualization, P.P., Y.G., and C.K.; methodology, P.P., Y.G., and C.K.; software, P.P. and Y.G.; validation, P.P., Y.G., and C.K.; formal analysis, P.P., Y.G., and C.K.; investigation, P.P., Y.G., and C.K.; data curation, P.P. and Y.G.; writing-original draft, P.P., Y.G., and C.K.; writing - review and editing, P.P., Y.G., and C.K.; visualization, P.P. and Y.G.; supervision, C.K.; project administration, C.K. All authors read and approved the final manuscript.

\section{Funding}

The results published here are in whole or part based upon data generated by The Cancer Genome Atlas (dbGaP accession phs000178) managed by the NCl and NHGRI. Information about TCGA can be found at http://cancergenome. nih.gov. This research is funded in part by the Gordon and Betty Moore Foundation's Data-Driven Discovery Initiative through Grant GBMF4554 to C.K. and by the US National Institutes of Health (R01GM122935). Also, the Advanced Scientific Computing Research (ASCR) program within the Office of Science of the DOE under contract number DE-AC02-05CH1 1231, the Exascale Computing Project (17-SC-20-SC), a collaborative effort of the US Department of Energy Office of Science and the National Nuclear Security Administration.

\section{Availability of data and materials}

Our implementation of VariantStore and all necessary scripts and output logs are available at Github [61]: https://github. com/Kingsford-Group/variantstore under BSD-2-Clause License. The code and scripts are also available at Zenodo [62]: (DOI: 10.5281/zenodo.5083774). The 1000 Genomes data used for the analyses described in this manuscript were obtained from http://ftp.1000genomes.ebi.ac.uk/vol1/ftp/release/20130502/. The TCGA data used for analyses described in this manuscript were obtained from https://portal.gdc.cancer.gov/. All the accession files used to download data are available at https://github.com/Kingsford-Group/variantstore/tree/master/eval_data_records/gdc_manifest. The script used to download the TCGA data is available at https://github.com/Kingsford-Group/variantstore/blob/master/scripts/ download.sh. The human reference data used for the analyses described in this manuscript were obtained from https:// hgdownload.soe.ucsc.edu/downloads.html\#human.

\section{Declarations}

Ethics approval and consent to participate

Not applicable

\section{Consent for publication}

Not applicable

\section{Competing interests}

C.K. is a co-founder of Ocean Genomics, Inc.

Received: 19 November 2020 Accepted: 27 July 2021

Published online: 19 August 2021

\section{References}

1. 1000 Genomes Project Consortium. A map of human genome variation from population-scale sequencing. Nature. 2010;467(7319):1061.

2. 1000 Genomes Project Consortium. An integrated map of genetic variation from 1,092 human genomes. Nature. 2012;491(7422):56.

3. 1000 Genomes Project Consortium. A global reference for human genetic variation. Nature. 2015;526(7571):68.

4. Lonsdale J, Thomas J, Salvatore M, Phillips R, Lo E, Shad S, Hasz R, Walters G, Garcia F, Young N. The genotype-tissue expression (GTEx) project. Nat Genet. 2013;45(6):580-5.

5. TCGA: The Cancer Genome Atlas Program. https://www.cancer.gov/about-nci/organization/ccg/research/ structural-genomics/tcga. Accessed Aug 2019.

6. Choudhury A, Ramsay M, Hazelhurst S, Aron S, Bardien S, Botha G, Chimusa ER, Christoffels A, Gamieldien J, Sefid-Dashti M. Whole-genome sequencing for an enhanced understanding of genetic variation among South Africans. Nat Commun. 2017:8(1):2062.

7. Roskosch S, Jónsson H, Björnsson E, Beyter D, Eggertsson HP, Sulem P, Stefánsson K, Halldórsson BV, Kehr B. PopDel identifies medium-size deletions jointly in tens of thousands of genomes. bioRxiv. 2019740225. http://dx. doi.org/10.1038/s41467-020-20850-5.

8. Groza C, Kwan T, Soranzo N, Pastinen T, Bourque G. Personalized and graph genomes reveal missing signal in epigenomic data. bioRxiv. 2019457101. http://dx.doi.org/10.1186/s13059-020-02038-8.

9. Albert FW, Kruglyak L. The role of regulatory variation in complex traits and disease. Nat Rev Genet. 2015;16(4): $197-212$. 
10. Eilbeck K, Quinlan A, Yandell M. Settling the score: variant prioritization and Mendelian disease. Nat Rev Genet. 2017;18(10):599-612.

11. Carvalho CM, Lupski JR. Mechanisms underlying structural variant formation in genomic disorders. Nat Rev Genet. 2016;17(4):224-38.

12. Kelleher J, Wong Y, Wohns AW, Fadil C, Albers PK, McVean G. Inferring whole-genome histories in large population datasets. Nat Genet. 2019;51(9):1330-8.

13. The Variant Call Format (VCF) Version 4.1 Specification. https://samtools.github.io/hts-specs/NCFv4.1.pdf. Accessed Mar 2019.

14. Ballouz S, Dobin A, Gillis JA. Is it time to change the reference genome? Genome Biol. 2019;20(1):1-9.

15. Laydon DJ, Bangham CR, Asquith B. Estimating T-cell repertoire diversity: limitations of classical estimators and a new approach. Philos Trans R Soc B Biol Sci. 2015;370(1675):20140291.

16. Brodin J, Krishnamoorthy M, Athreya G, Fischer W, Hraber P, Gleasner C, Green L, Korber B, Leitner T. A multiple-alignment based primer design algorithm for genetically highly variable DNA targets. BMC Bioinformatics. 2013;14(1):255.

17. Kanduri C, Bock C, Gundersen S, Hovig E, Sandve GK. Colocalization analyses of genomic elements: approaches, recommendations and challenges. Bioinformatics. 2019;35(9):1615-24.

18. The Computational Pan-Genomics Consortium. Computational pan-genomics: status, promises and challenges. Brief Bioinform. 2018;19(1):118-35.

19. Bayer PE, Golicz AA, Scheben A, Batley J, Edwards D. Plant pan-genomes are the new reference. Nat Plants. 2020;6: 914-20.

20. Sheikhizadeh S, Schranz ME, Akdel M, de Ridder D, Smit S. Pantools: representation, storage and exploration of pan-genomic data. Bioinformatics. 2016;32(17):487-93.

21. Tonkin-Hill G, MacAlasdair N, Ruis C, Weimann A, Horesh G, Lees JA, Gladstone RA, Lo S, Beaudoin C, Floto RA, et al. Producing polished prokaryotic pangenomes with the Panaroo pipeline. Genome Biol. 2020;21(1):1-21.

22. Rand KD, Grytten I, Nederbragt AJ, Storvik GO, Glad IK, Sandve GK. Coordinates and intervals in graph-based reference genomes. BMC Bioinformatics. 2017;18(1):263.

23. Ballouz S, Dobin A, Gillis JA. Is it time to change the reference genome? Genome Biol. 2019;20(1):1-9.

24. Garrison E, Sirén J, Novak AM, Hickey G, Eizenga JM, Dawson ET, Jones W, Garg S, Markello C, Lin MF, Paten B, Durbin R. Variation graph toolkit improves read mapping by representing genetic variation in the reference. Nat Biotechnol. 2018;36:875-9.

25. Hein J. A new method that simultaneously aligns and reconstructs ancestral sequences for any number of homologous sequences, when the phylogeny is given. Mol Biol Evol. 1989;6(6):649-68.

26. Lee C, Grasso C, Sharlow MF. Multiple sequence alignment using partial order graphs. Bioinformatics. 2002;18(3): 452-64.

27. Dilthey A, Cox C, Iqbal Z, Nelson MR, McVean G. Improved genome inference in the MHC using a population reference graph. Nat Genet. 2015;47(6):682-8.

28. Eggertsson HP, Jonsson H, Kristmundsdottir S, Hjartarson E, Kehr B, Masson G, Zink F, Hjorleifsson KE, Jonasdottir A, Jonasdottir A. Graphtyper enables population-scale genotyping using pangenome graphs. Nat Genet. 2017:49(11):1654-60.

29. Kim D, Paggi JM, Park C, Bennett C, Salzberg SL. Graph-based genome alignment and genotyping with HISAT2 and HISAT-genotype. Nat Biotechnol. 2019;37(8):907-15.

30. Rakocevic G, Semenyuk V, Lee W-P, Spencer J, Browning J, Johnson IJ, Arsenijevic V, Nadj J, Ghose K, Suciu MC. Fast and accurate genomic analyses using genome graphs. Nat Genet. 2019;51:354-62.

31. Rautiainen M, Mäkinen V, Marschall T. Bit-parallel sequence-to-graph alignment. Bioinformatics. 2019;35(19): 3599-607.

32. Sirén J. Indexing variation graphs. In: 2017 Proceedings of the Ninteenth Workshop on Algorithm Engineering and Experiments (ALENEX). SIAM; 2017. p. 13-27. https://doi.org/10.1137/1.9781611974768.2.

33. Sirén J, Garrison E, Novak AM, Paten B, Durbin R. Haplotype-aware graph indexes. Bioinformatics. 2020;36(2):400-7.

34. Layer RM, Kindlon N, Karczewski KJ, Quinlan AR, Consortium EA. Efficient genotype compression and analysis of large genetic-variation data sets. Nat Methods. 2016;13(1):63-5.

35. Li H. BGT: efficient and flexible genotype query across many samples. Bioinformatics. 2015;32(4):590-2.

36. Danek A, Deorowicz S. GTC: how to maintain huge genotype collections in a compressed form. Bioinformatics. 2018;34(11):1834-40.

37. Zheng X, Gogarten SM, Lawrence M, Stilp A, Conomos MP, Weir BS, Laurie C, Levine D. SeqArray-a storage-efficient high-performance data format for WGS variant calls. Bioinformatics. 2017;33(15):2251-7.

38. Brookes AJ, Robinson PN. Human genotype-phenotype databases: aims, challenges and opportunities. Nat Rev Genet. 2015;16(12):702-15.

39. Kutzera J, May P. Variant-DB: a tool for efficiently exploring millions of human genetic variants and their annotations. In: International Conference on Data Integration in the Life Sciences. Springer; 2017. p. 22-8. https://doi.org/10. 1007/978-3-319-69751-2_3.

40. Vandeweyer G, Van Laer L, Loeys B, Van den Bulcke T, Kooy RF. VariantDB: a flexible annotation and filtering portal for next generation sequencing data. Genome Med. 2014;6(10):74.

41. Variation Graph Toolkit. https://github.com/vgteam/vg. Accessed Mar 2019.

42. IGSR: The International Genome Sample Resource. http://www.internationalgenome.org/home. Accessed Mar 2019.

43. BCF Toolkit. https://samtools.github.io/bcftools/bcftools.html. Accessed Mar 2019.

44. GenoTypes Compressor. https://github.com/refresh-bio/GTC. Accessed Jan 2020.

45. Martiniano R, Garrison E, Jones ER, Manica A, Durbin R. Removing reference bias and improving indel calling in ancient dna data analysis by mapping to a sequence variation graph. BioRxiv. 2020782755. http://dx.doi.org/10. 1186/s13059-020-02160-7. 
46. Groza C, Kwan T, Soranzo N, Pastinen T, Bourque G. Personalized and graph genomes reveal missing signal in epigenomic data. Genome Biol. 2020;21(1):1-22.

47. Hickey G, Heller D, Monlong J, Sibbesen JA, Sirén J, Eizenga J, Dawson ET, Garrison E, Novak AM, Paten B. Genotyping structural variants in pangenome graphs using the vg toolkit. Genome Biol. 2020;21(1):1-17.

48. Chen S, Krusche P, Dolzhenko E, Sherman RM, Petrovski R, Schlesinger F, Kirsche M, Bentley DR, Schatz MC, Sedlazeck FJ, et al. Paragraph: a graph-based structural variant genotyper for short-read sequence data. Genome Biol. 2019;20(1):1-13.

49. Li H. Minimap2: pairwise alignment for nucleotide sequences. Bioinformatics. 2018;34(18):3094-100

50. Samtools. http://www.htslib.org/. Accessed Mar 2021.

51. Garrison E, Marth G. Haplotype-based variant detection from short-read sequencing. arXiv preprint arXiv:1207.3907. 2012. https://arxiv.org/abs/1207.3907.

52. O'Neil P, Cheng E, Gawlick D, O'Neil E. The log-structured merge-tree (LSM-tree). Acta Informatica. 1996;33(4): $351-85$.

53. Gog S, Beller T, Moffat A, Petri M. From theory to practice: plug and play with succinct data structures. In: 13th International Symposium on Experimental Algorithms, (SEA 2014); 2014. p. 326-37.

54. Pandey P, Bender MA, Johnson R, Patro R. A general-purpose counting filter: making every bit count. In: Proceedings of the 2017 ACM International Conference on Management of Data. ACM; 2017. p. 775-87. https://doi. org/10.1145/3035918.3035963.

55. SDSL: Succinct Data Structure Library. https://github.com/simongog/sdsl-sdsllite. Accessed Mar 2019.

56. Almodaresi F, Pandey P, Patro R. Rainbowfish: a succinct colored de Bruijn graph representation. In: 17 th International Workshop on Algorithms in Bioinformatics (WABI 2017); 2017. Schloss Dagstuhl-Leibniz-Zentrum fuer Informatik.

57. Pandey P, Almodaresi F, Bender MA, Ferdman M, Johnson R, Patro R. Mantis: a fast, small, and exact large-scale sequence-search index. Cell Syst. 2018;7(2):201-7.

58. Almodaresi F, Pandey P, Ferdman M, Johnson R, Patro R. An efficient, scalable and exact representation of high-dimensional color information enabled via de Bruijn graph search. In: International Conference on Research in Computational Molecular Biology. Springer; 2019. p. 1-18. https://doi.org/10.1007/978-3-030-17083-7_1.

59. Almodaresi F, Pandey P, Ferdman M, Johnson R, Patro R. An efficient, scalable, and exact representation of high-dimensional color information enabled using de Bruijn graph search. J Comput Biol. 2020;27(4):485-99. https://doi.org/10.1089/cmb.2019.0322.

60. Raman R, Raman V, Satti SR. Succinct indexable dictionaries with applications to encoding k-ary trees, prefix sums and multisets. ACM Trans Algoritm(TALG). 2007;3(4):43.

61. VariantStore: an index for large-scale genomic variant search. https://github.com/Kingsford-Group/variantstore. Accessed Mar 2020

62. Pandey P, Gao Y, Kingsford C. Variantstore: an index for large-scale genomic variant search. 2021. https://doi.org/10. 5281/zenodo.5083774. Accessed July 2021.

\section{Publisher's Note}

Springer Nature remains neutral with regard to jurisdictional claims in published maps and institutional affiliations.

\section{Ready to submit your research? Choose BMC and benefit from:}

- fast, convenient online submission

- thorough peer review by experienced researchers in your field

- rapid publication on acceptance

- support for research data, including large and complex data types

- gold Open Access which fosters wider collaboration and increased citations

- maximum visibility for your research: over $100 \mathrm{M}$ website views per year

At BMC, research is always in progress.

Learn more biomedcentral.com/submissions 